\title{
Control design and experimental validation for flexible multi-body systems pre-compensated by inverse shapers
}

\author{
Dan Pilbauer ${ }^{\mathrm{a}, \mathrm{b}}$, Wim Michiels ${ }^{\mathrm{b}}$, Jaroslav Bušek ${ }^{\mathrm{a}}$, David Osta ${ }^{\mathrm{a}}$, Tomáš \\ Vyhlídal $^{\mathrm{a}}$ \\ ${ }^{a}$ Dept. of Instrumentation and Control Eng., Faculty of Mechanical Engineering, and Czech \\ Institute of Informatics, Robotics and Cybernetics - CIIRC, Czech Technical University in \\ Prague, Technická 4, 16607 Praha 6, Czech Republic \\ ${ }^{b}$ Dept. of Computer Science, KU Leuven, Celestijnenlaan 200A, B-3001 Heverlee, Belgium
}

\begin{abstract}
A complex methodology for control of flexible multi-body systems is proposed with the objective to achieve a favorable distribution of system motion so that the oscillatory mode of the flexible part is not excited. As the key element, the recently proposed concept of a feedback loop with an inverse distributed delay shaper is adopted. Unlike in existing works, the mutual coupling between the primary (controlled) structure and secondary (flexible) structure of oscillatory nature is explicitly taken into account in the controller design. First, an easy to apply method to isolate the flexible mode to be targeted in the shaper design is proposed. Secondly, the interconnection of the system, shaper and the controller is formulated as a set of delay differential algebraic equations. Then the spectral optimization control design technique is applied to achieve fast dynamics of the infinite dimensional system. The viability of the overall methodology is validated by both simulations and experiments in an extensive case study example.
\end{abstract}

Keywords: time-delay, inverse shapers, vibration control, spectral optimization, spectral abscissa

\section{Introduction}

Input shaping, a well known technique for compensating undesirable oscillatory modes of mechanical systems based on signal shapers with time-delays was firstly published in 1957 by O.J. Smith who introduced the posicast filter [1]. Subsequently, extensive studies have been made by Singer, Seering and Singhose [2, 3] who proposed zero-vibration (ZV), zero-vibration-derivative (ZVD) and extra-insensitive (EI) shapers. Further extension of the shapers for multi-modes flexible structures have for example been proposed in [4, 5] and with a discrete version in [6]. A typical application of input shaping is pre-compensation of payload oscillations suspended at a crane trolley, [7, 8]. For an extensive review on input shaping over last 50 years, see [9]. 
Next to the classical feed-forward arrangement of the shapers which can only handle the effect of the reference command, there was an impulse to place shapers in a feedback interconnection in order to eliminate the effect of unmeasurable disturbances on the excitation of the flexible modes. This technique was analyzed already by Smith in [10], where he developed a basic scheme with a compensator and a shaper in the feedback. However, it is important to mention that, as shown in [11], the application of the proposed scheme is applicably if and only if both the controller and the system are bi-proper as their inversion is needed in the compensator. Subsequently a direct augmentation of the standard feedback loop with a pre-tuned input shaper was proposed in [12]. A further investigation of the system and stability of the closed loop using the root locus was done in [13] and [14]. It was shown that placing the signal shaper in the loop between the controller and the system is not effective in the objective of suppressing the vibration caused by disturbance variables, except the sensor disturbance.

Following the preliminary results in [15], a novel feedback loop architecture was proposed in [11], along with both simulation and experimental verification. See also [16] for the double mode compensation task. This method is based on including an inverse shaper into the feedback loop. As has been reported, by the shaper inversion in the feedback interconnection, the high frequency zeros of the shaper are turned into the closed-loop poles. This fact eliminates applicability of classical shapers with the lumped delays to this task as their spectrum is neutral with the high frequency zeros lying either at or close to the imaginary axis. Instead, new types of shapers based on distributed delays need to be applied [17, 18, 19], resulting in a relatively safe retarded distribution of the closed-loop poles. As studied in detail in [11], favorable properties of the shaper in distributing the closed-loop responses in time so that the oscillatory mode is not excited are transferred to the closed-loop system if and only if a sufficiently fast controller is applied. Too slow dynamics of the closed loop may result in disruption of the 'notch' characteristics brought by the shaper.

To position approaches using inverse shapers in a broader context, let us point to article [23], where a scheme with an inverse shaper was compared with a classical control scheme, where the desired 'notch' filter characteristic at the flexible mode frequency was achieved by the controller. The following advantages of the architecture with an inverse shaper were revealed: i) achieving the mode compensation in the channels from both reference and disturbance signals, which cannot be achieved by a classical scheme, ii) more favorable responses of the primary structure - in the shaper free scheme the mode compensation was achieved at the cost of considerably larger overshoots.

Another point that needs to be mentioned is that in the scheme with the inverse shaper applied in [11, 15, 16], it was assumed that there is no backward coupling from the the flexible system part to the controlled main body. Recently, it was shown in [20] that when the mutual coupling between these two subsystems takes place, a special attention needs to be paid to deriving the mode to which the shaper needs to be tuned. Note that as a rule, this mode is not present in the overall system dynamics. To determine the mode 
a method has been proposed in [20] based on an input-output transformation of the multi-body system into the controlled subsystem, the attached residual oscillatory dynamics, and an internal feedback loop representing the dynamical coupling. The transformation eliminating the system input is performed over the time domain system equations. The oscillatory mode to be targeted is determined as complex eigenvalue of the reduced order flexible system.

Even though the benefit of including an inverse shaper to the feedback in order to pre-compensate oscillations induced by both set-point changes and disturbances [11, 20], the control design of arising infinite dimensional system has not been properly addressed. As documented in [11], for simple structure systems, no special attention needs to be paid to the controller design as including the inverse shaper to the loop does not affect the closed-loop characteristics substantially. Only a number of rules needs to be followed when designing the PID controllers. However, extension of this rather heuristic approach towards coupled multi-body systems for which controllers of higher complexity are needed is problematic. In this case, the infinite dimensionality of the closed loop brought by the input shaper needs to be taken into account.

The first contribution of this paper consists of a transfer function based method for determining the target oscillatory mode of the systems based on transfer function approach, which is easier to apply compared to relatively complex methodology presented in [20]. As the second, key contribution, a modified closed-loop scheme with an inverse shaper and a fixed order controller is proposed to control efficiently multi-body flexible systems. In order to fulfill the requirement on the fast dynamics of the closed-loop system, the spectrum is optimization using the method of [21], yet as the presented control design framework is generic, other characteristics such as $H_{\infty}$ criteria can be optimized as well. The work follows preliminary results presented in [22], where however, the coupling between the primary and secondary structure was not considered.

For the controller design by the spectral method, the model of Multi-Degree Of Freedom (MDOF) system will be interconnected with an inverse shaper forming the overall model in Differential Algebraic Equations (DDAEs), which are amendable for describing interconnected systems. The controller will then be obtained by minimization of the spectral abscissa, the real part of the rightmost eigenvalue, as a function of the controller parameters. This procedure has originally been proposed in 24] for retarded systems, and extended to DDAEs in [21], including a MATLAB too 1 . Finally, note that the overall control scheme can be considered as a delay-based controller. Currently, there is an increasing interest on this class of controllers due to their practical implications 25, 26].

The paper is organized as follows. First, preliminaries on input shaping are given in Section 2 together with inverse application of the shaper in the feedback loop. Then, a method for decoupling is presented in Section 3 together with the introduction of the modified control scheme with an inverse shaper. In Section 4 , the model with an inverse shaper is formulated as a set of delay differential

\footnotetext{
${ }^{1}$ Available from: http://twr.cs.kuleuven.be/research/software/delay-control/
} 
algebraic equations and the spectrum optimization based control design method is adjusted for this specific task. Then, a thorough case study application follows in Section 5 , where all the proposed techniques are validated by both simulations and experiments. Finally, the Conclusions are given in Section 6 .

\section{Preliminaries on input shaper and its application in feedback loop}

The objective of applying an inverse shaper in a feedback interconnection with a system is to fully or partially compensate oscillatory modes of a secondary (typically flexible) structure. The mode has to remain unexcited either by a setpoint change or by external disturbances. Before outlining the key properties of the scheme with an inverse shaper, let us briefly recall fundamental properties of input shaping.

A general description of a delay based input shaper is as follows,

$$
v(t)=a w(t)+(1-a) \int_{0}^{T} w(t-\mu) d h(\mu),
$$

where $w$ and $v$ are the shaper input and output, respectively, $a \in \mathbb{R}^{+}, a<1$ is the gain parameter, and the distribution of the delays is prescribed by the nondecreasing function $h(\mu)$, with length $T$. Note that in the classical input shapers $[3,4,10]$, the lumped delays with step-wise response are applied. Alternatively recently proposed shapers [17, 19] include distributed delays.

The transfer function of the shaper (1) is given by

$$
S(s)=a+(1-a) F(s, T),
$$

where $F(s, T)=\mathcal{L}\left\{\int_{0}^{T} w(t-\mu) d h(\mu)\right\}$ is the Laplace transform of delay. The shaper is designed in a way that its transfer function contains only zeros, some of which play crucial role in compensating the oscillatory modes of the system linked to the shaper in feed-forward manner. The simplest zero-vibration (ZV) shaper is for example designed so that its dominant zeros cancel the oscillatory poles [2, 10]. Denoting the mode to be compensated by $r_{1,2}=-\zeta \omega \pm \omega \sqrt{1-\zeta^{2}}$, where $\omega$ is the natural frequency of oscillations and $\zeta$ is the damping, the shaper parameters are tuned to achieve $S\left(r_{1}\right)=0$. An enhanced robustness in mode compensation can be achieved by a shaper of higher complexity known as EI (extra insensitive) shaper [27], see also references therein, where the design is done by optimizing the residual vibration characteristics. The feedback interconnection with the inverse shaper proposed in [20] is shown in Fig. [1. If the shaper $S(s)$ is tuned to compensate the oscillatory mode of $G(s)$ (e.g. by zero-pole cancellation for ZV), this property has a potential to be transferred to all the above transfer functions $T_{y_{s} r}(s)=G(s) \frac{C(s) H(s) S(s)}{S(s)+C(s) H(s)}, T_{y_{s} d_{1}}(s)=G(s) \frac{H(s) S(s)}{S(s)+C(s) H(s)}$ and $T_{y_{s} d_{2}}(s)=G(s) \frac{1 S(s)}{S(s)+C(s) H(s)}$, because $S(s)$ appears in their numerators. Thus, the oscillatory mode is precompensated not only in the responses induced by the reference $r$, but also in the responses induced by the disturbances $d_{1}, d_{2}$. 


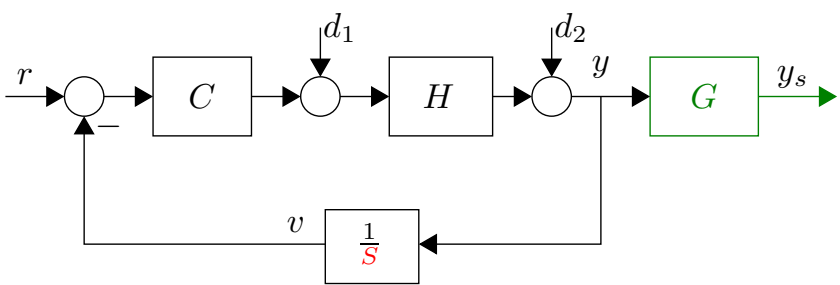

Figure 1: Feedback interconnection of an inverse shaper with no coupling between the system body and flexible structure [1]

\subsection{Distributed zero-vibration shaper and its inverse form}

In this work, we apply a distributed-zero-vibration (DZV) shaper (see [17] for more details), with transfer function

$$
S(s)=a+(1-a) \frac{1-e^{-s T}}{T s} e^{-s \tau} .
$$

The shaper is parametrized by the lengths of the distributed $T$ and the lumped $\tau$ delay, and by the gain $a<1$. The parameters are tuned in order to compensate the target mode $r_{1,2}=-\zeta \omega \pm \omega \sqrt{1-\zeta^{2}}$ of the flexible system $G(s)$. As derived in Lemma 1 of 19], selecting the length of the distributed delay $T \in\left(0, \frac{\pi}{\omega \sqrt{1-\zeta^{2}}}\right]$, this leads to

$$
\tau=\frac{\pi+\varphi}{\omega \sqrt{1-\omega^{2}}}, a=\frac{m \mathrm{e}^{\frac{\zeta}{\sqrt{1-\zeta^{2}}}(\pi+\varphi)}}{1+m \mathrm{e}^{\frac{\zeta}{\zeta \sqrt{1-\zeta^{2}}}(\pi+\varphi)}},
$$

where $m=\left|\bar{G}\left(-\omega \zeta+j \omega \sqrt{1-\zeta^{2}}, T\right)\right|$ and $\varphi=\arg \left(\bar{G}\left(-\omega \zeta+j \omega \sqrt{1-\zeta^{2}}, T\right)\right)$ with $\bar{G}(s, T)=\frac{1-e^{-s T}}{T s}$. Concerning the inverse shaper implementation, the transfer function $\frac{1}{S(s)}$ can be turned to the time domain representation

$$
\begin{array}{r}
v(t)=\frac{1}{a}(y(t)-(1-a) z(t)), \\
\dot{z}(t)=\frac{1}{T}(v(t-\tau)-v(t-(\tau+T))),
\end{array}
$$

where $y, v, z$ are input, output and internal state of the inverse shaper. It should be mentioned that this transformation results in additional dynamics, characterized by the introduction of an eigenvalue at zero [28].

\section{Virtual decoupling of the flexible structure and modified control scheme with inverse shaper}

Compared to [1], we assume mutual coupling between the dynamics of the main, primary structure and the secondary structure. This type of coupling does 
not fit into the classical schemes in Fig. 1, which is based on the simplifying assumptions that the dynamics of the primary structure $H(s)$ are not affected by the secondary structure $G(s)$ and that the control input acts on the flexible output only through the system output. As revealed in [20] for the systems with coupled dynamics, as a rule, the mode to be targeted by the input shaper does not appear in the system spectrum and needs to be isolated. In this section, we first provide a transfer function candidate representing the flexible structure to be pre-compensated. Then we validate the functionality of modified scheme with inverse shaper parametrized to damp the oscillatory mode of the flexible transfer function candidate.

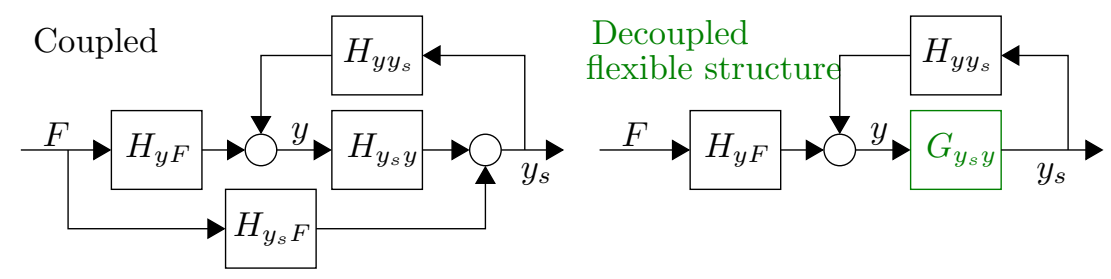

Figure 2: Isolation of the flexible structure from the coupled system.

Assume the physical model of the coupled system is given by the two linear higher order differential equations

$$
\begin{aligned}
& \sum_{k=0}^{n_{y}} a_{1, k} y^{(k)}=\sum_{k=0}^{m_{f, 1}} b_{1, k} F^{(k)}+\sum_{k=0}^{m_{y_{s}}} c_{1, k} y_{s}^{(k)}, \\
& \sum_{k=0}^{n_{y_{s}}} a_{2, k} y_{s}^{(k)}=\sum_{k=0}^{m_{f, 2}} b_{2, k} F^{(k)}+\sum_{k=0}^{m_{y}} c_{2, k} y^{(k)},
\end{aligned}
$$

where $F, y$ and $y_{s}$ represent the input, controlled output, and output of the flexible structure. The causality of the physical relations impose the following inequalities: $n_{y} \geq m_{f, 1}, n_{y} \geq m_{y_{s}}, n_{y_{s}} \geq m_{f, 2}$ and $n_{y_{s}} \geq m_{y}$. The above system of equation can be turned to the representation in the transfer function form

$$
\begin{aligned}
y(s) & =H_{y F}(s) F(s)+H_{y y_{s}}(s) y_{s}(s), \\
y_{s}(s) & =H_{y_{s} F}(s) F(s)+H_{y_{s} y}(s) y(s) .
\end{aligned}
$$

with the scheme given in Fig. 2 - top. As can be seen, the flexible output $y_{s}$ is influenced not only by the primary structure output $y$, but also by the system input $F$ via a path bypassing the output. As a candidate of the transfer function the mode of which should be targeted by the shaper, let the transfer function $G_{y_{s} y}(s)=\frac{y_{s}(s)}{y(s)}$ be derived covering also the bypass path. The idea of eliminating the bypass path is shown in bottom figure of Fig. 2 , 


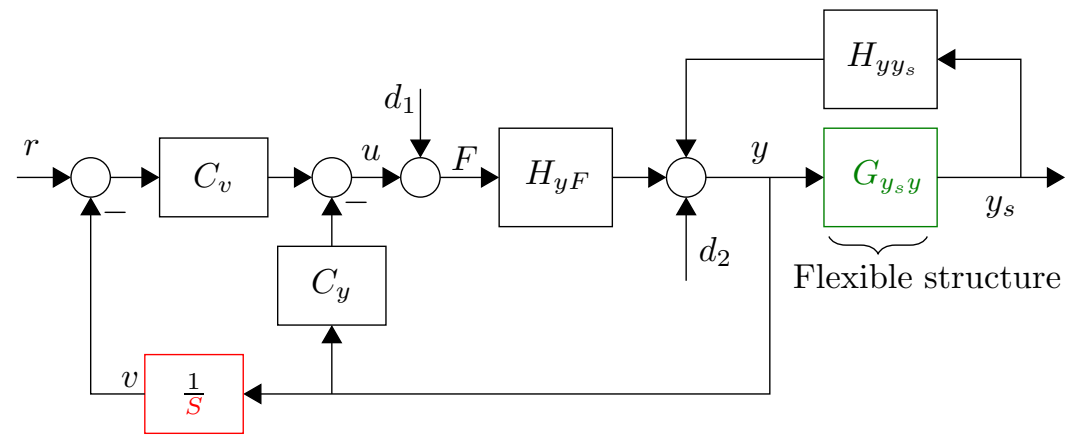

Inverse shaper

Figure 3: Modified scheme with the inverse shaper to control multi-body flexible systems

Expressing $F(s)$ from (8) and substituting it to (10), we obtain

$$
y_{s}(s)=H_{y_{s} F}(s) \frac{1}{H_{y F}(s)}\left(y(s)-H_{y y_{s}}(s) y(s)\right)+H_{y_{s} y}(s) y(s),
$$

from which the transfer function of the flexible part is determined by

$$
G_{y_{s} y}(s)=\frac{y_{s}(s)}{y(s)}=\frac{H_{y_{s} y}(s) H_{y F}(s)+H_{y_{s} F}(s)}{H_{y_{s} F}(s) H_{y y_{s}}(s)+H_{y F}(s)} .
$$

Let us note that if the system input $F$ does not influence $y_{s}$ directly, i.e. the structural link from $F$ to $y_{s}$ is just via $y$ and thus $H_{y_{s} F}(s)=0$, the derivation of the flexible transfer function reduces to $G_{y_{s} y}(s)=H_{y_{s} y}(s)$.

In the second step, it will be checked whether the flexible mode of $G_{y_{s} y}(s)$ is pre-compensated by the inverse shaper in all the closed-loop channels, analogously as it holds for the scheme in Fig. 1. To analyze this we first include the controllers, consisting of controller $C_{v}(s)$ processing a signal passing via the inverse shaper, an additional controller $C_{y}(s)$ included with unshaped feedback directly from the control output $y$.

Examining the channels from reference $r$ to flexible output $y_{s}$

$$
T_{y_{s} r}(s)=G_{y_{s} y}(s) \frac{S(s) C_{v}(s) H_{y F}(s)}{S(s)\left(1+H_{y F}(s) C_{y}(s)-H_{y y_{s}}(s) G_{y y_{s}}(s)\right)+H_{y F}(s) C_{v}(s)},
$$

from input disturbance $d_{1}$ to flexible output $y_{s}$

$$
T_{y_{s} d_{1}}(s)=G_{y_{s} y}(s) \frac{S(s) H_{y F}(s)}{S(s)\left(1+H_{y F}(s) C_{y}(s)-H_{y y_{s}}(s) G_{y y_{s}}(s)\right)+H_{y F}(s) C_{v}(s)},
$$


and from output disturbance $d_{2}$ to flexible output $y_{s}$

$$
T_{y_{s} d_{2}}(s)=G_{y_{s} y}(s) \frac{S(s)}{S(s)\left(1+H_{y F}(s) C_{y}(s)-H_{y y_{s}}(s) G_{y y_{s}}(s)\right)+H_{y F}(s) C_{v}(s)},
$$

it can be seen that required zero-pole cancellation between $S(s)$ and $G_{y_{s} y}(s)$ can be projected to all the channels (12), (13) and (14) as the shaper transfer function $S(s)$ appears in numerators of the transfer functions $T_{y_{s}(\cdot)}$. Thus, if the shaper is tuned to the flexible mode of $G_{y_{s} y}(s)$, the mode compensation can take a place in all the considered closed-loop channels.

However, the prompt control action is needed to follow the time-distribution of the responses brought by the input shaper. This requirement on rather aggressive controller $C_{v}(s)$ can be seen directly from the denominators of (12)(14). In particular it needs to be achieved that the following inequality $\mid 1+$ $H_{y F}(s) C_{y}(s)-H_{y y_{s}}(s) G_{y y_{s}}(s)|>>| H_{y F}\left(r_{1}\right) C_{v}\left(r_{1}\right) \mid$, where $r_{1}$ is the flexible pole to be compensated, does not hold. If this happens, the zero of $T_{y(\cdot)}(s)=$ $T_{y_{S}(\cdot)}(s) \frac{1}{G_{y_{s} y}(s)}$ brought by the shaper $S(s)$ is compensated fully or partially by the pole of $T_{y(\cdot)}(s)$ which lies close to the dominant zero of $S(s)$.

To sum up, the zeros of $S(s)$ should be tuned to compensate the poles of transfer function $G_{y_{s} y}(s)$, which describes the dynamics of the secondary structure as if it were detached from the overall system.

\section{Controller design by spectral method}

Analogously to the scheme in Fig. 1] considered in [11], in order to achieve active mode compensation by the shaper in all of the channels (12), (13) and (14), the prompt control action is needed to follow the time-distribution of the responses brought by the input shaper. In order to meet the requirement on the rather aggressive controller, we apply spectrum optimization based approach proposed in 21] for time delay systems formulated as a set of differential algebraic equations. The method is based on minimizing the spectral abscissa, defined as the real part of the rightmost root. By shifting the abscissa to the left, the response speed is being increased. Thus, the objective of this control design is to set the controller parameters so that the fastest possible responses are achieved. Before describing the controller tuning design task in more detail, we formulate the shaper-system interconnection in the DDAE standard form.

\subsection{Reformulation into the DDAE standard form}

The interconnection of the mechanical model and the inverse shaper is modelled using Delay Differential Algebraic Equations (DDAEs), also called descriptor system. DDAE description allows to include algebraic equations of

the inverse shaper in state-space representation, as well as delayed parts of the shaper. The DDAE description will be later used in a design of the controller. 
Considering the closed-loop system according to Fig. 3, the DDAE description will consists of a system

$$
\dot{\boldsymbol{x}}(t)=\boldsymbol{A} \boldsymbol{x}(t)+\boldsymbol{B} u(t),
$$

in the classical state space form, where $u=F$, and the inverse DZV shaper given by (4)-(5). Note that for the control design task, the disturbances are considered zero. Thus, the DDAE description of the system can be formulated as

$$
\left\{\begin{aligned}
\dot{\boldsymbol{x}}(t) & =\boldsymbol{A} \boldsymbol{x}(t)+\boldsymbol{B} u(t), \\
0 & =-v(t)+\frac{1}{a}(y(t)-(1-a) z(t)), \\
\dot{z}(t) & =\frac{1}{T}(v(t-\tau)-v(t-(\tau+T)))
\end{aligned}\right.
$$

with new state vector $\boldsymbol{z}=\left[\begin{array}{lll}\boldsymbol{x}^{T} & v & z\end{array}\right]^{T}$ and the output given by

$$
\boldsymbol{Y}(t)=(y(t) \quad v(t))^{T} .
$$

\subsection{Controller structure and design}

In order to stabilize and optimize the spectrum of the resulting infinite dimensional system, following the methodology proposed in [21] we consider a fixed order dynamic feedback controller in the form

$$
K:\left\{\begin{array}{ccc}
\dot{\boldsymbol{x}}_{K}(t) & =\boldsymbol{A}_{K} \boldsymbol{x}_{K}(t)+\boldsymbol{B}_{K} \boldsymbol{Y}(t), \\
u(t) & =\boldsymbol{C}_{K} \boldsymbol{x}_{K}(t)+\boldsymbol{D}_{K} \boldsymbol{Y}(t),
\end{array}\right.
$$

with $k$ denoting order of the controller. Considering the defined system output vector (17), the overall control scheme is assumed as in Fig. 3 with

$$
\left[\begin{array}{l}
C_{y}(s) \\
C_{v}(s)
\end{array}\right]=\boldsymbol{C}_{K}\left(s \boldsymbol{I}-\boldsymbol{A}_{K}\right)^{-1} \boldsymbol{B}_{K}+\boldsymbol{D}_{K}
$$

Note that the spectrum optimization for time delay systems has originally been proposed as a tool to design parameters of state feedback controller $u(t)=$ $\boldsymbol{K} \boldsymbol{x}(t)$ [24], see also 29] with preceding continuous pole placement method and [30] with a direct pole placement method and application to a heat exchanger control. As motivated in 21], the given controller structure in a form of a dynamical system (18) provides larger degree of freedom compared to the state feedback controller, which is crucial particularly for the time delay systems with infinite spectrum. Besides, since the feedback is taken from the output, no state observer is needed.

In the spectrum optimization of the closed-loop system two issues deserve special attention. First, the current design technique [21] does not provide control over the zeros distribution. This however is not a limiting factor at all, if the control scheme is turned to a two-degree of freedom scheme often applied in industrial applications 31]. This aspect will be addressed in more detail in the case study application in the next section. Second, due to the presence of the shaper the modes of the flexible structure become uncontrollable. This motivates a controller design based on minimizing the modified spectral abscissa,

$$
c(K):=\sup \left\{\Re(\lambda): s \in \Sigma(K) \backslash\left\{r_{1}, r_{2}\right\}\right\},
$$


as a function of the elements of matrices $\left(\boldsymbol{A}_{K}, \boldsymbol{B}_{K}, \boldsymbol{C}_{K}, \boldsymbol{D}_{K}\right)$. For this task we use the algorithm and software described in [21], which has been adapted in removing the non-physical eigenvalues and the modes of the secondary structure, from the eigenvalue computation.

It is important to note that the function $K \mapsto c(K)$ is in general not everywhere differentiable, even not everywhere Lipschitz continuous, even though the dependence of the system matrices of the closed-loop system on the controller parameters is smooth. To tackle these challenges, the core optimization tasks in the method of [21] are done using the software HANSO (Hybrid Algorithm for Nonsmooth Optiization) [32]. The underlying algorithm only requires the evaluation of the objective function along with its derivatives with respect to the controller parameters, whenever the objective function is differentiable. These are obtained from the computation of the rightmost eigenvalues and associated eigenvectors.

\subsection{Summary of the design procedure}

The control design procedure with the objective to pre-compensate the oscillatory mode of the flexible part in all the channels can be summarized as follows:

1. Isolate the oscillatory mode $r_{1,2}$ to be compensated by virtual decoupling the flexible part as proposed in Section 3, or by applying the approach of [20]. $r_{1,2}$ is given as the dominant oscillatory mode of $G_{y_{s} y}(s)$ (11).

2. Select an input shaper with a distributed delay, e.g. DZV (2), and parametrize it to the mode $r_{1,2}$ (by (3) for DZV).

3. Form the DDAE representation (16) given as an interconnection of the system (15) and the implementation of the shaper inversion.

4. Select an order of the controller (18) considering the closed-loop structure given in Fig. (3).

5. Parametrize the controller of the arising interconnected systems with time delays, e.g. by minimizing the modified spectral abscissa (20) as proposed in [21] and highlighted in Subsection 4.2.

\section{Case study}

The system under consideration with the scheme depicted in Fig. 4 consist of a series of two carts connected via spring and damper and a pendulum attached to the cart 2. The control action force $F$ interacts with the cart 1 whereas the position of the cart 2 , denoted by $x_{2}$, is the controlled variable. The control objective is to propose a control scheme and design the controller for adjusting the position $y=x_{2}$ so that the oscillatory mode of the cart-pendulum interconnection is not excited. 


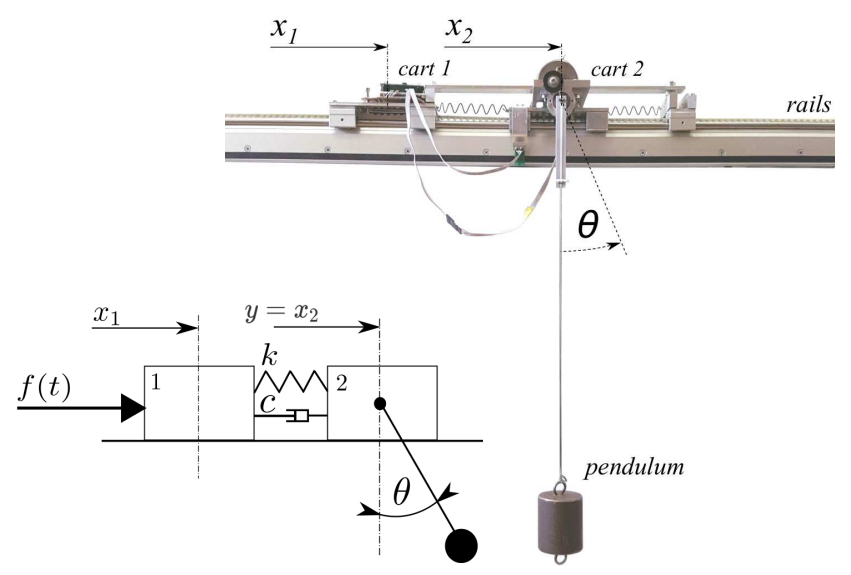

Figure 4: Photo of the laboratory set-up and a scheme of its mechanical configuration

\subsection{Experimental set-up}

The laboratory set-up built according to the scheme in Fig. 4 is shown in Fig. 4. The frame is built of the standardized aluminum profiles. Carts 1 and 2 connected each to the other by a spring slide on linear ball-bearing rails. A pendulum is connected to the cart 2. Cart 1 is fixed to a belt underneath which is powered by an AC servo drive with an axial transmission. The drive is controlled by an industrial control unit (ESTUN - Pronet-E-04A) commanded by analog signal from an attached PC with MATLAB, Simulink and Real Time Windows Target. The control unit is operated in the torque-generator mode the generated torque and thus the force $F$ is proportional to the analog control signal. The position of the cart is measured by an incremental rotary sensor located in the actuator shaft. The pendulum angle is measured by a magnetic angular sensor fixed to a pendulum shaft which is nested in bearings.

\subsection{Model of the three degree of freedom system}

The linearized physical model of the set-up is given by the following three cross-coupled second order differential equations

$$
\begin{gathered}
m_{1} \ddot{x}_{1}(t)+\mu_{1} \dot{x}_{1}(t)=k\left(x_{2}(t)-x_{1}(t)\right)+c\left(\dot{x}_{2}(t)-\dot{x}_{1}(t)\right)+F(t), \\
\left(m_{2}+m_{a}\right) \ddot{x}_{2}(t)+\mu_{2} \dot{x}_{2}(t)=k\left(x_{1}(t)-x_{2}(t)\right)+c\left(\dot{x}_{1}(t)-\dot{x}_{2}(t)\right)-m_{a} l \ddot{\theta}(t), \\
m_{a} l \ddot{\theta}(t)+m_{a} g \theta(t)=-m_{a} \ddot{x}_{2}(t)
\end{gathered}
$$

with parameters of the primary structure given as masses $m_{1}=1.8 \mathrm{~kg}, m_{2}=$ $3.25 \mathrm{~kg}$, resistance coefficients $\mu_{1}=14.5 \mathrm{~kg} . \mathrm{s}^{-1}, \mu_{2}=3 \mathrm{~kg} . \mathrm{s}^{-1}$, spring stiffness $k=300 N \cdot \mathrm{m}^{-1}$ and damping $c=1 \mathrm{~kg} \cdot \mathrm{s}^{-1}$. The parameters of the secondary structure are given by the mass of the bob $m_{a}=2 \mathrm{~kg}$ and pendulum length $l_{a}=0.67 \mathrm{~m}$. Notice that the ideal pendulum model is considered as oscillation damping is minimal and can be neglected. Note that some of the parameters 
have been measured, such as masses and pendulum length, while the others have been identified experimentally based on a series of pulse responses.

As a first step we bring (21) into a state-space representation of the form

$$
\left\{\begin{array}{c}
\dot{\boldsymbol{x}}(t)=\boldsymbol{A} \boldsymbol{x}(t)+\boldsymbol{B} u(t) \\
y(t)=\boldsymbol{C} \boldsymbol{x}(t)
\end{array}\right.
$$

with matrices $\boldsymbol{A} \in R^{6 \times 6}, \boldsymbol{B} \in R^{6}$ and $\boldsymbol{C} \in R^{1 \times 6}$, with state vector $\boldsymbol{x}=$ $\left[x_{1}, \dot{x}_{1}, x_{2}, \dot{x}_{2}, \theta, \dot{\theta}\right]^{T}$, input $u(t)=F(t)$ and output $y(t)=x_{2}(t)$.

\subsection{Isolation of the flexible mode and shaper parametrization}

As the first step, the target flexible mode to be compensated by the shaper needs to be isolated. Considering zero initial conditions and applying the Laplace transform, system (21) can be transformed to

$$
\begin{gathered}
M_{x_{1}}(s) x_{1}(s)=N_{x}(s) x_{2}(s)+F(s), \\
M_{x_{2}}(s) x_{2}(s)=N_{x}(s) x_{1}(s)+N_{\theta}(s) \theta(s), \\
M_{\theta}(s) \theta(s)=N_{x_{2}}(s) x_{2}(s),
\end{gathered}
$$

where $M_{x_{1}}=m_{1} s^{2}+\left(\mu_{1}+c\right) s+k, M_{x_{2}}=\left(m_{2}+m_{a}\right) s^{2}+\left(\mu_{2}+c\right) s+k$, $M_{\theta}=l s^{2}+g, N_{x}(s)=c s+k, N_{\theta}(s)=-m_{a} l s^{2}$ and $N_{x_{2}}(s)=-s^{2}$. Eliminating the internal variable $x_{1}(s)$ the system can be turned to the transfer function form (8)-(10)

$$
\begin{gathered}
x_{2}(s)=\frac{1}{M_{x_{1}}(s) M_{x_{2}}(s)-N_{x}(s)^{2}} F(s)+\frac{N_{\theta}(s) M_{x_{1}}(s)}{M_{x_{1}}(s) M_{x_{2}}(s)-N_{x}(s)^{2}} \theta(s), \\
\theta(s)=\frac{N_{x_{2}}(s)}{M_{\theta}(s)} x_{2}(s) .
\end{gathered}
$$

Comparing (10) with (25), we have $H_{y_{s F}}(s)=\frac{\theta(s)}{F(s)}=0$, resulting to the reduction of the flexible transfer function (11) to

$$
G_{y_{s} y}(s)=\frac{N_{x_{2}}(s)}{M_{\theta}(s)}
$$

Thus, the target mode for the shaper is determined by the mode of the ideal pendulum $r_{1,2}= \pm j \omega$, where $\omega=\sqrt{\frac{g}{l}}=3.83 \mathrm{~s}^{-1}$.

To parametrize the shaper (2), according to Lemma 1 of [19], the following parameters $a=0.474, \tau=0.616 \mathrm{~s}$ are obtained, with the selection $T=\frac{\pi}{2 \omega}=$ $0.474 s$.

\subsection{Controller design by spectrum optimization}

The first step of the controller design by [21] is formulation of the overall DDAE description of the system pre-compensated by the inverse shaper. In order to achieve entire asymptotic elimination of the control error $e=r-v$ in the responses to the disturbances, an extra integrator is included to the relevant 
feedback loop of system (22). By this choice, the system order is increased by one. Including also the inverse shaper (4)-(5), the DDAE form is given by

$$
\left\{\begin{aligned}
\dot{\mathbf{x}}(t) & =\boldsymbol{A} \mathbf{x}(t)+\boldsymbol{B} u(t), \\
\dot{q}(t) & =v(t)-r(t) \\
\dot{z}(t) & =\frac{1}{T}(v(t-\tau)-v(t-(\tau+T))) . \\
0 & =-v(t)+\frac{1}{a}\left(x_{2}(t)-(1-a) z(t)\right) .
\end{aligned}\right.
$$

With the generalized state

$$
\boldsymbol{X}(t)=\left[x_{1}(t) \dot{x}_{1}(t) x_{2}(t) \dot{x}_{2}(t) \theta(t) \dot{\theta}(t) q(t) z(t) v(t)\right]^{T},
$$

and with outputs selected as the positions of the primary structure $x_{2}$ together with the output of the inverse shaper

$$
\boldsymbol{Y}(t)=\left[x_{2}(t) q(t)\right]^{T} .
$$

Equations (27)-(29) can be rewritten into a compact form

$$
\left\{\begin{array}{l}
\mathcal{E} \dot{\boldsymbol{X}}(t)=\mathcal{A}_{0} \boldsymbol{X}(t)+\mathcal{A}_{1} \boldsymbol{X}(t-\tau)+\mathcal{A}_{2} \boldsymbol{X}(t-2 \tau)+\mathcal{B} u(t) \\
\boldsymbol{Y}(t)=\mathcal{C} \boldsymbol{X}(t)
\end{array}\right.
$$

In the subsequent step in the control system design, the control loop structure in Fig. 3 with the controller (18) is considered. As described above, the optimization based approach [21] is applied to minimize the modified spectral abscissa $c(K)$. In order to achieve as fast dynamics as possible, the controller order has been gradually increased. For each of the order setting the algorithm has been run repeatedly many times with random initial conditions. Balancing the system order size and the value of $c(K)$ minimum achieved, the best results have been achieved for the controller order $k=6$, for which $\min (c(K))=-2.022 s^{-1}$.

The results of the optimization procedure can be seen in Fig. 5. Next to the final spectrum of the closed-loop system (black dots), the spectra of original system (blue $\times$ ) and the input shaper (red o) are shown. Notice that the spectrum of the original system truly does not involve the oscillatory mode that needs to be targeted by the shaper (denoted as blue + ). Notice also, that this mode is compensated by the right-most couple of the input shaper zeros. In the shaper spectrum, we can also see a beneficial retarded distribution with the roots departing to the left as their moduli increase. In agreement with the analysis performed in [11], this retarded character is beneficially transferred to the closed-loop poles with high moduli. This applies also for high frequency poles not seen in the figure. On the other hand, it is nicely seen that the closedloop poles of low moduli have been shifted to left and are formed closely to the left of the minimized spectral abscissa. 2

Transforming the controller (18) to the transfer function form (19), we obtain

\footnotetext{
${ }^{2}$ For the controller application in Matlab, the polynomial coefficients are considered of double precision.
} 


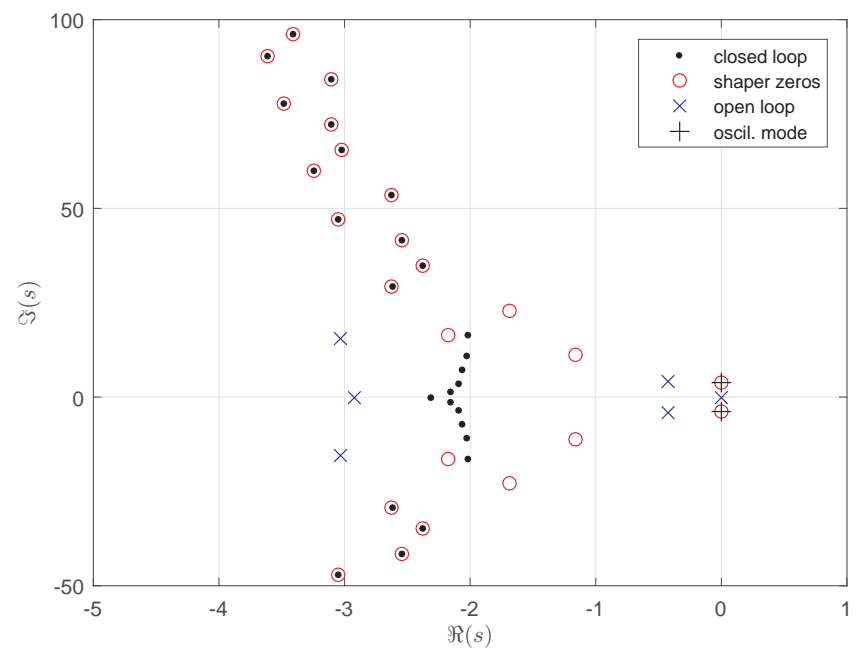

Figure 5: Rightmost spectra of the original system (blue $\times$ ), input shaper (red $\circ$ ), closed-loop system (black dots). The oscillatory mode that needs to be targeted by the shaper denoted as black + .

$$
\begin{gathered}
C_{y}(s)=\frac{-26.72 s^{6}-437.5 s^{5}+5246 s^{4}+1.731 e 5 s^{3}+1.789 e 6 s^{2}+1.267 e 7 s+2.75 e 7}{s^{6}+54.71 s^{5}+1395 s^{4}+2.054 e 4 s^{3}+1.861 e 05 s^{2}+1.065 e 6 s+3.457 e 6}, \\
C_{v}(s)=\frac{78.38 s^{6}-3125 s^{5}-3.383 e 04 s^{4}+3.487 e 4 s^{3}+1.433 e 6 s^{2}-1.308 e 6 s+2.085 e 7}{s^{6}+54.71 s^{5}+1395 s^{4}+2.054 e 4 s^{3}+1.861 e 5 s^{2}+1.065 e 6 s+3.457 e 6},
\end{gathered}
$$

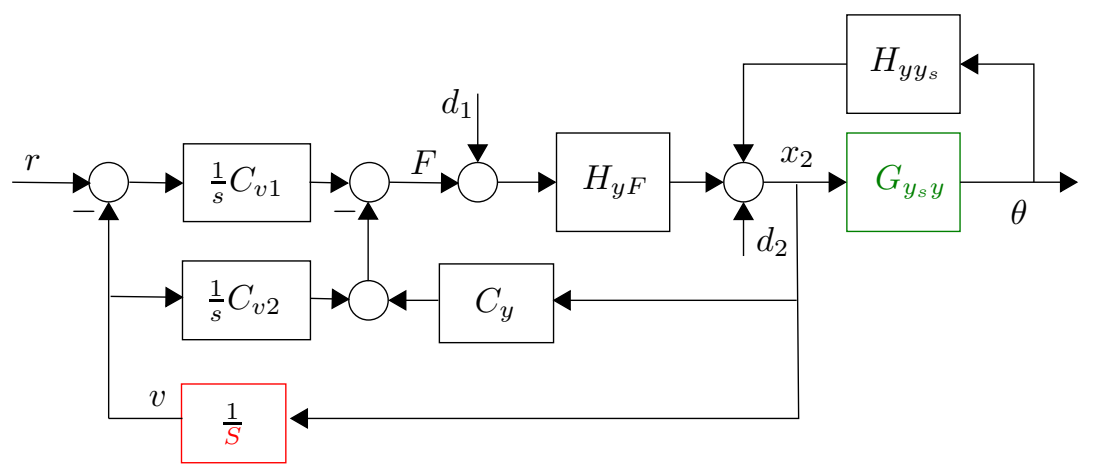

Figure 6: Two degree of freedom control scheme with the inverse shaper and fixed-order controllers

Due to the negative signs of some of the coefficients at the numerator of $C_{v}(s)$, it is clear that the controller has positive zeros which will become zeros of the closed-loop transfer function $T_{y_{s}, r}(s)$ given in (12). This would result 
in non-minimum phase characteristics with known undesirable consequences for the responses from the reference $r$.

In order to remove this insufficiency, which in fact can be expected whenever the method proposed in 21] is applied as the position of the controller zeros is left free, the control design scheme Fig. 3 has been turned to the two-degree of freedom structure in Fig. 6. The key benefit of this scheme widely acknowledged in industrial applications is the possibility to tune the set-point tracking responses by freely adjusting the controller zeros.

As can be seen in Fig. 6. the controller $C_{v}(s)$ has been decomposed to $C_{v 1}(s)$ in the feed-forward part and to $C_{v 2}(s)$ in the feedback part of the closed loop satisfying

$$
C_{v}(s)=C_{v 1}(s)+C_{v 2}(s) .
$$

Let us recall that an additional integrator has been included to (27) which now appears in a serial connection with both $C_{v 1}(s)$ and $C_{v 2}(s)$, forming the controllers to be finally implemented.

Due to an additional integrator in a series with $C_{v 1}(s)$ in the scheme in Fig. 6. the following choice

$$
C_{v 1}(s)=\frac{c\left(s-z_{1}\right)\left(s-z_{2}\right)}{(s-p)} .
$$

leads to an overall controller of PID form. Consequently, $C_{v 2}(s)$ is obtained directly from (33). The tuning of the set-point response $T_{y r}(s)$ can then be done by the controller zeros $z_{1}, z_{2}$ and the pole of the filter $p$. Concerning the gain $c$, the choice $c=\lim _{s \rightarrow 0} C_{v}(s) \frac{\left|z_{1} z_{2}\right|}{|p|}$ is to retain the static gain in the whole path from $v$ equal to the designed controller $C_{v}(s)$.

\subsection{Simulation and experimental results}

First, the performance of the designed control scheme has been validated by simulations in Matlab Simulink (ode45 method, Relative tolerance 1e-6) with the results shown in Fig. 7 in the form of step responses to output $d_{1}$ and input $d_{2}$ disturbances and the reference $r$. The given responses with minimal overshoot in the reference step response have been achieved for the controller setting $z_{1,2}=-3 s^{-1}$ and $p=-20 s^{-1}$. Note that by increasing the magnitude of the zeros, faster response could be achieved, but at the cost of higher overshoot.

The validation starts with rather unrealistic output disturbance, which can be interpreted as instant shifting of cart 2 in time $\Delta t \rightarrow 0$. As can be seen, the oscillatory mode has been fully compensated. The pendulum motion stops as soon as the disturbance is rejected, no residual oscillations occur afterward. The same conclusion confirming the active compensation of the mode can be made also for the subsequent input disturbance by an additional force acting at the cart 1. Finally, the set-point response is presented. Even though the shaper is placed to the feedback in the inverse form, it can clearly be seen that the controlled variable follows the time-distribution corresponding to the shaper as it was included in the feed-forward manner. Also in this case, the 

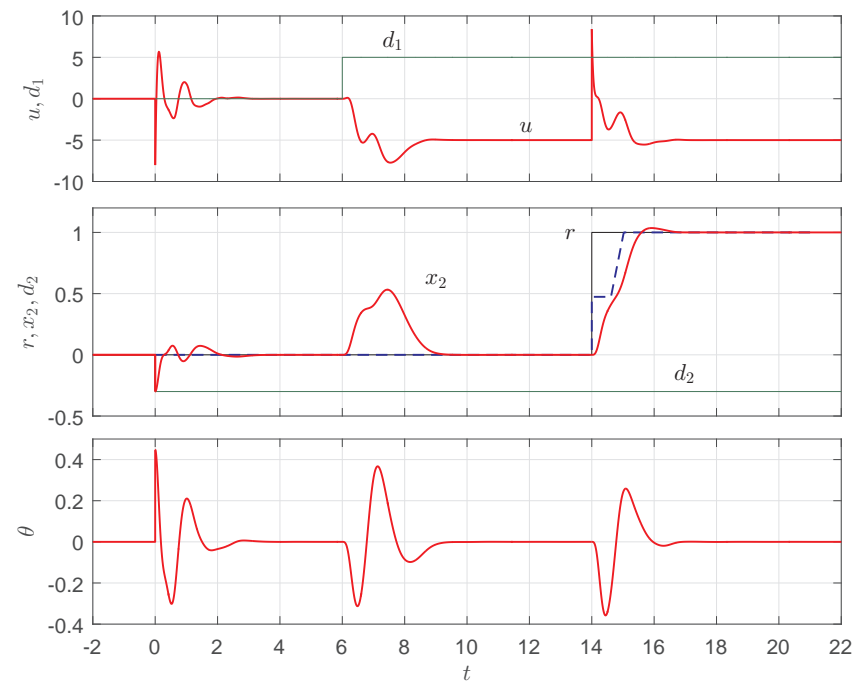

Figure 7: Simulation results - responses to the step changes of i) output disturbance $d_{2}$ at $t=0 s$, ii) input disturbance $d_{1}$ at $t=6 s$ and iii) reference $r$ at $t=14 \mathrm{~s}$ for which also a shaped signal is shown by dashed line.

entire compensation of the oscillatory mode has been achieved. To sum up, the simulations have successfully validated functioning of the proposed control scheme with the inverse shaper.

As the main result of this section, we present the experimental validation of the proposed control scheme. First, a set-point response is shown in Fig. 8 - left. For comparison, the results of experiment are accompanied with results of simulation. As can be seen, almost ideal compensation has been achieved. The controlled variable has only slightly larger overshoot compared to the ideal simulated response. This consequently results in larger amplitudes in the pendulum oscillations. However, minimal residual vibrations can be seen after the control transient is finished, i.e. after time $t=188 \mathrm{~s}$. Let us note that slight differences between the measured and simulated responses can be accounted to the non-linearities, friction in particular.

Analogous results confirming the functionality of the proposed scheme in the disturbance rejection are shown in Fig. 8 -right. The disturbance of rather input nature was achieved be deflecting manually the position of the cart 1 and holding it in the shifted position for approximately two seconds. Due to the integration character of the controller $C_{v 1}(s)$, holding of the cart in approximately fixed position caused increase of the control signal $u$ representing the action force. After releasing the cart, the controllers drive the position of the cart 2 to the original position in a shaped manner so that almost none residual vibrations occur. Thus, to sum up, the functioning of the scheme has also been confirmed 

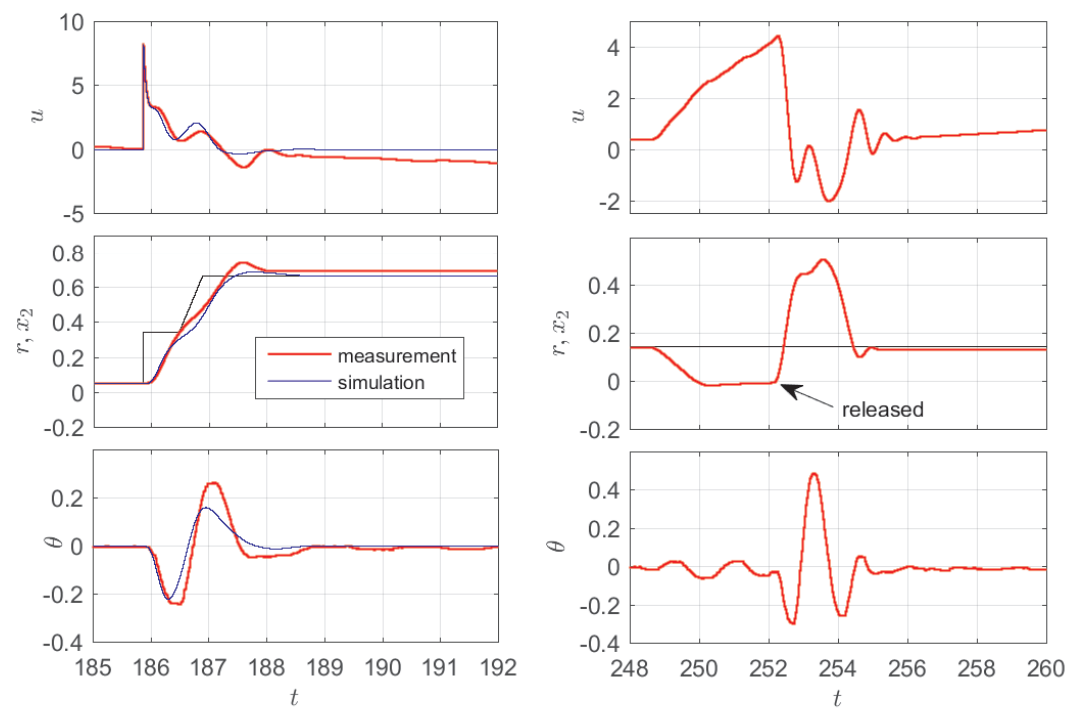

Figure 8: Experimental validation of the scheme in Fig. 6 Left - set-point response. Right disturbance rejection

by the experiment ${ }^{3}$.

\section{Conclusions}

The main contribution of this paper is design and validation of an overall scheme for control of multi-body system pre-compensated by inverse shapers. The preliminary results on control design by spectrum optimization presented in 22] have been extended to the class of more complex multi-body dynamics with coupling between the main body and flexible part. In the first step, the flexible dynamics is decomposed from the model by applying transfer function based method. A thorough analysis of the properties of the inverse shaper in the systems with coupled dynamics was provided. The system with the inverse shaper is translated to the descriptor state space model for which dynamical fixed-order controller is introduced. The paper describes how to design the parameters of the controller using spectral abscissa optimization technique modified in order to handle system with the inverse shaper and uncontrollable eigenvalues.

With the case study, we demonstrated the application of the inverted DZV shaper in the feedback loop using the proposed technique. In order to minimize the response time, a dynamic controller is introduced. The results show how

${ }^{3}$ The video from the experiment can be seen at
http://control.fs.cvut.cz/en/aclab/experiments/invshaper


considering coupling is essential for the design of the inverse shaper in order to ensure stability and desired dynamics for the close loop. The fixed-order controller, designed by direct optimization technique, can provide sufficient control actions with very limited number of measured outputs. The results are thoroughly validated by both simulations and experiments.

\section{Acknowledgement}

The presented research has been supported by the Czech Science Foundation under the project 16-17398S. This work was also supported by the Programme of Interuniversity Attraction Poles of the Belgian Federal Science Policy Office (IAP P6-DYSCO), by OPTEC, the Optimization in Engineering Center of the KU Leuven, by project G0A5317N of the Research Foundation-Flanders (FWO - Vlaanderen), and by the project UCoCoS, funded by the European Unions Horizon 2020 research and innovation programme under the Marie SklodowskaCurie Grant Agreement No 675080.

\section{References}

[1] O. J. Smith, Posicast control of damped oscillatory systems, Proceedings of the IRE 45 (9) (1957) 1249-1255. doi:10.1109/JRPROC.1957.278530.

[2] N. C. Singer, W. P. Seering, Preshaping command inputs to reduce system vibration, Journal of dynamic systems, measurement, and control 112 (1) (1990) 76-82.

[3] W. Singhose, W. Seering, N. Singer, Residual vibration reduction using vector diagrams to generate shaped inputs, Journal of Mechanical Design 116 (2) (1994) 654-659. doi:10.1115/1.2919428.

[4] Y.-G. Sung, W. Singhose, Robustness analysis of input shaping commands for two-mode flexible systems, Control Theory \& Applications, IET 3 (6) (2009) 722-730. doi:10.1049/iet-cta.2007.0328

[5] T. D. Tuttle, W. P. Seering, A zero-placement technique for designing shaped inputs to suppress multiple-mode vibration, in: American Control Conference, 1994, Vol. 3, IEEE, 1994, pp. 2533-2537. doi:10.1109/ACC.1994.735015.

[6] M. O. Cole, A discrete-time approach to impulse-based adaptive input shaping for motion control without residual vibration, Automatica 47 (11) (2011) 2504-2510. doi:10.1016/j . automatica.2011.08.039.

[7] J. Vaughan, D. Kim, W. Singhose, Control of tower cranes with doublependulum payload dynamics, IEEE Transactions on Control Systems Technology 18 (6) (2010) 1345-1358. 
[8] W. Singhose, D. Kim, M. Kenison, Input shaping control of doublependulum bridge crane oscillations, Journal of Dynamic Systems, Measurement, and Control 130 (3) (2008) 034504.

[9] W. Singhose, Command shaping for flexible systems: A review of the first 50 years, International Journal of Precision Engineering and Manufacturing 10 (4) (2009) 153-168.

[10] O. J. Smith, Feedback control systems, New York: McGraw-Hill Book Co., Inc., (1958) pp. 331-345.

[11] T. Vyhlídal, M. Hromčík, V. Kučera, M. Anderle, On feedback architectures with zero vibration signal shapers, IEEE Transactions on Automatic Controdoi:10.1109/TAC.2015.2492502.

[12] J. Y. Hung, Feedback control with posicast, Industrial Electronics, IEEE Transactions on 50 (1) (2003) 94-99. doi:10.1109/TIE.2002.804979.

[13] J. R. Huey, W. Singhose, Trends in the stability properties of clss controllers: a root-locus analysis, Control Systems Technology, IEEE Transactions on 18 (5) (2010) 1044-1056. doi:10.1109/TCST.2009.2031681.

[14] U. Staehlin, T. Singh, Design of closed-loop input shaping controllers, in: American Control Conference, 2003. Proceedings of the 2003, Vol. 6, IEEE, 2003, pp. 5167-5172. doi:10.1109/ACC.2003.1242547.

[15] T. Vyhlídal, M. Hromčík, V. Kučera, Inverse signal shapers in effective feedback architecture, in: Proc. European Control Conference, IEEE, 2013, pp. 4418-4423.

[16] T. Vyhlídal, M. Hromčík, V. Kučera, M. Anderle, Double oscillatory mode compensation by inverse signal shaper with distributed delays, in: Control Conference (ECC), 2014 European, IEEE, 2014, pp. 1121-1126. doi:10.1109/ECC.2014.6862561.

[17] T. Vyhlídal, V. Kučera, M. Hromčík, Signal shaper with a distributed delay: Spectral analysis and design, Automatica 49 (11) (2013) 3484-3489. doi:10.1016/j.automatica.2013.08.029

[18] T. Vyhlídal, V. Kučera, M. Hromčík, Zero vibration shapers with distributed delays of various types, in: Decision and Control (CDC), 2013 IEEE 52nd Annual Conference on, IEEE, 2013, pp. 940-945. doi:10.1109/CDC.2013.6760003.

[19] T. Vyhlídal, M. Hromčík, Parameterization of input shapers with delays of various distribution, Automatica 59 (2015) 256 - 263. doi:doi:10.1016/j.automatica.2015.06.025.

[20] M. Hromcik, T. Vyhlidal, Inverse feedback shapers for coupled multibody systems, IEEE Transactions on Automatic Control 62 (9) (2017) 48044810 . 
[21] W. Michiels, Spectrum-based stability analysis and stabilisation of systems described by delay differential algebraic equations, IET control theory \& applications 5 (16) (2011) 1829-1842. doi:10.1049/iet-cta.2010.0752.

[22] D. Pilbauer, T. Vyhlídal, W. Michiels, Spectral design of output feedback controllers for systems pre-compensated by input shapers, in: Proceedings of the 12th IFAC Workshop on Time-Delay Systems, 2015, pp. 1-6. doi:10.1016/j.ifacol.2015.09.363

[23] D. Pilbauer, W. Michiels, T. Vyhlídal, A comparison of shaper-based and shaper-free architectures for feedforward compensation of flexible modes, Accepted in: Valmorbida G., Seuret A., Sipahi R., Boussaada I. (Eds.), bookseries: Advances in Delays and Dynamics, Delays and interconnections: Methodology, algorithms and applications, Springer. Available as TW672 Report, Department of Computer Science, KU Leuven.

[24] S. Vandewalle, W. Michiels, K. Verheyden, J. Vanbiervliet, A nonsmooth optimisation approach for the stabilisation of time-delay systems, ESAIM: Control, Optimisation and Calculus of Variations 14 (3) (2008) 478-493. doi:10.1051/cocv:2007060

[25] A. Ramírez, S. Mondié, R. Garrido, R. Sipahi, Design of proportionalintegral-retarded (pir) controllers for second-order lti systems, IEEE Transactions on Automatic Control 61 (6) (2016) 1688-1693.

[26] I. Boussaada, I.-C. Morărescu, S.-I. Niculescu, Inverted pendulum stabilization: Characterization of codimension-three triple zero bifurcation via multiple delayed proportional gains, Systems \& Control Letters 82 (2015) $1-9$.

[27] J. Vaughan, A. Yano, W. Singhose, Comparison of robust input shapers, Journal of Sound and Vibration 315 (4) (2008) 797-815.

[28] D. Pilbauer, W. Michiels, T. Vyhlídal, Distributed delay input shaper design by optimizing smooth kernel functions, Journal of the Franklin Institute.

[29] W. Michiels, D. Roose, An eigenvalue based approach for the robust stabilization of linear time-delay systems, International Journal of Control 76 (7) (2003) 678-686.

[30] W. Michiels, T. Vyhlídal, P. Zítek, Control design for time-delay systems based on quasi-direct pole placement, Journal of Process Control 20 (3) (2010) 337-343.

[31] L. Kevickzy, C. Bányász, Two-degree-of-freedom control systems: The Youla parameterization approach, Academic Press, 2015.

[32] M. Overton, Hanso: a hybrid algorithm for nonsmooth optimization, Available from cs. nyu. edu/overton/software/hanso. 